Marc van den Berg*

\title{
National Library of the Netherlands on Transformation
}

https://doi.org/10.1515/bfp-2018-0065

\begin{abstract}
After a short introduction to the Koninklijke Bibliotheek (KB), the article deals with the question, why The National Library of The Netherlands (KB) needed to transform. Major challenges are discussed: e.g. new roles for KB in the public library domain and the more general transformation towards a digital business. Next, the article describes strategy: how to transform, following both internal and external drivers? At the end a short overview of current status and results are given.
\end{abstract}

Keywords: National Library; Netherlands; digital transformation; strategy

\section{Die Nationalbibliothek der Niederlande in Transformation}

Zusammenfassung: Nach einer kurzen Zusammenfassung über die Koninklijke Bibliotheek (KB) beschäftigt sich der Artikel mit der Frage, warum die Nationalbibliothek der Niederlande eine Transformation benötigt. Folgende wichtigen Neuerungen werden diskutiert: die neue Rolle der KB für den öffentlichen Sektor sowie eine ganz generelle Transformation zu einem digitalen Wandel. Danach erfolgt eine Beschreibung, wie dieser Wandel vollzogen werden soll. Welche internen und welche externen Einflüsse spielen hierbei eine Rolle? Gegen Ende des Artikels wird zusammenfassend ein kurzer Überblick über den bisherigen Stand und die Ergebnisse der Transformation gegeben.

Schlüsselwörter: Nationalbibliothek; Niederlande; Digitale Transformation; Strategie

A modern day book is a physical copy of a digital original. Metadata can lead you to many other things than the original object is was created for.

The KB welcomes 100,000 visitors to its premises each year.

Given these kind of observations, it becomes clear that a national library, dealing with the written word, needs to adapt to a world that is becoming more and more 'digital'.

*Kontaktperson: Marc van den Berg, Marc.vandenBerg@KB.nl
In case of the National Library of the Netherlands (KB), adaptation was not enough, we needed to transform and therefore we started a transition process, which has not ended yet (and probably never will).

\section{KB: Short introduction}

\section{KB mission}

The Koninklijke Bibliotheek in Den Haag is the National Library of the Netherlands. ${ }^{1}$ Driven by the power of the written word we further intellectual development, proficiency and creativity in the Netherlands. To this end, we seek collaboration with partners in the domains of public libraries, cultural heritage and academics. The KB promotes the visibility, usability and longevity of the Dutch Library Collection, defined as the collective holdings of all publicly funded libraries in the Netherlands. Unhindered access to these collections furthers the development of new ideas and allows researchers to build upon the ideas of their predecessors.

KB serves three domains: (1) cultural heritage, with mass digitization programs, large print and e-collections and an active role in the Dutch Digital Heritage network; (2) research, with an active role in the (digital) humanities and social sciences disciplines e.g. with our KB-Lab and by providing access to our collections, both physically and electronically (including data services); (3) the public library domain where $\mathrm{KB}$ is responsible for national programmes and themes since 2015, an innovation agenda, the delivery of management information, research on e.g. societal impact, the provisioning of the national digital infrastructure for public libraries (authentication, data ware house, website-as-a-service, open search platform) and the purchase and delivery of e-content on a national scale (licensed material, e-books platform).

1 http://www.kb.nl/en. 


\section{$2 \mathrm{~KB}$ in numbers}

The KB was founded in 1798 and has an annual budget of $€ 89$ million; staff of 412 employees ( $350 \mathrm{fte}$ ) excl. temporal or external employees.

Holdings include

- 7 million items $=115 \mathrm{~km}$ of library materials (books, newspapers, journals, manuscripts, maps)

- 10,800 current periodicals; 500 licensed databases and e-journals and 13,000 licensed e-books

- $100 \mathrm{M}$ digitized pages

- $\quad 10,000$ archived websites

- digital storage \pm 1 petabyte

Our e-books service had 3,5 M loans during 2017.

\section{Reasons to transform}

Adapting to a world of information that has dominantly becoming digital is one thing, but the $\mathrm{KB}$ had more reasons to transform. Starting from 2015 the KB was expanded, jumping from \pm 250 to over 400 in staff and doubling its budget. This was the result from a merger with three organisations: two from the public library domain (BNL, a digital innovation project organisation and SIOB, the public library sector institute) and one organisation specialised in making Dutch literature (and context information) available online (DBNL, Digital Library for Dutch Literature). The four organisations that merged under the name 'KB, National Library of the Netherlands' were very different in structure, culture, and method.

Where the merger was a good enough reason to transform, so was the consequence that the KB from 2015 onward was responsible for the combined themes, programmes, projects and activities of the four merged organisations and for consolidating them, find synergy in them, streamline them, optimise them, in other words: being responsible for the life cycle management of a much bigger and more complex portfolio than any of the four organisations originally held. Altogether, the targeted customer group of the KB consist now of nearly all members of the Dutch population: from the very young - e.g. through the 'bookstart' programme - to the elderly - e.g. with large font- or audiobooks - and everybody else in between - e.g. with our discovery platform 'Delpher'.

Of course, adaptation to an information-driven world in which 'digital' is the norm, is a very strong persuader for (permanent) change. Together with our network partners KB is working towards a 'National Digital Library' in which digital collections from publicly funded organisations in the Netherlands are made available as free and as soon as possible to the world in all sorts of ways: as digital objects, as linked data, as data service. Sometimes KB will provide the discovery tools (like Delpher, DBNL, or one of the other thematic sites KB maintains, like 'Memory of the Netherlands'); sometimes the discovery tools are provided by one of our partners, or by search giants like Google. Working towards a National Digital Library means working as a link in several information 'chains' with various partners, some public, some commercial. This asks for the rethinking of processes and that challenges the organisation as a whole.

Like any organisation that is going through a 'digital transformation', KB is struggling with IT-infrastructure, both legacy and the 'hot \& new'. Also, 'dealing with data' (not to forget dealing with metadata) is becoming a real challenge, as is information/cyber security and being compliant with new privacy regulations.

\section{How to transform: KB strategy}

\subsection{Internal KB strategy}

Management of the KB soon realised that the kind of transformation needed to deal with the challenges mentioned above, meant playing on several boards simultaneously. Processes, methods, culture, structure, even behaviour and work atmosphere; each and everything was bound to be influenced and was needed to get the fundamental and lasting results we needed. To this end, our director general composed a 'transition scheme' that shows the different elements that need to be 'tuned' (hence the 'knobs' in the diagram) to make the transition work.

The five elements - culture, management (governance), strategy, knowledge \& competences and organisational structure ('OFP') - are all important and all needed, but the order and the amount in which to tune them can differ greatly over time and per specific challenge. The idea behind the diagram is that we will need to tune the knobs repeatedly until transition to the 'new KB' is complete, if it ever is. (For the biblically inspired: there is a Jerichoanalogy to be found here.)

Next, we looked at the positioning of the KB. We found that our positioning needed to reflect all of our broadened efforts in a single and strong statement. The new positioning we came up with - "Driven by the power of the written word, we work as a network organisation to contribute to a smart, competent and creative society" - is now core to our mission (see text box above). The new positioning is geared towards people, and the society they form, and encompasses reading for fun, for learning and for re- 


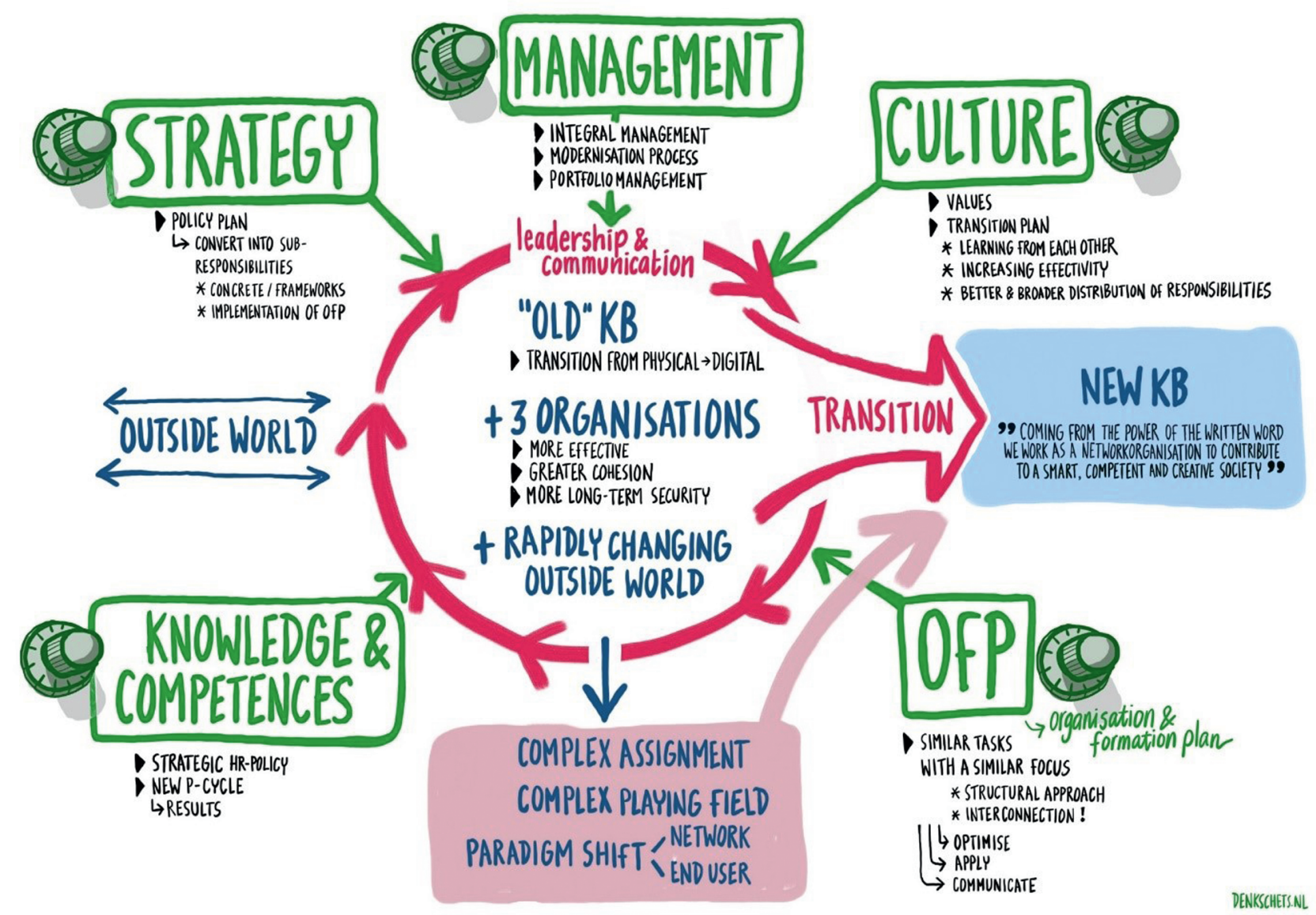

Abb. 1: Transition scheme of KB

search. The new positioning also helps us in determining our position in external relations and joint efforts.

We organised an organisation-wide selection process for new core values. Values that needed to reflect the new $\mathrm{KB}$ after the merger. We came up with four core values: the $\mathrm{KB}$ is reliable, innovative, connecting and open. The last value, 'open', is more an ambition; as a (national/research) library we can't offer complete openness for all content, so here our goal is to deliver as much content open to the public as we can. 'Open' can also be understood as 'transparent' and in that sense we truly want to be 'open'. We try to use these new core values in everything that we do and discover new uses for them all the time. E. g. recently we started using them in job interviews.

\subsection{External KB strategy}

Transforming the KB was also done by consequently putting the customer first. For an organisation that previously derived its raison d'être mainly from its role in collecting publications and supporting research, this was quite a change. With the massive and growing use of our online services, this was nevertheless an obvious route. We formulated six customer related objectives and used them as a measure stick for our strategy and for accepting or prioritising project proposals.

We realised that over time the KB had given rise to many initiatives that lack coherency, beautiful as each of them is. So we are undertaking a re-branding all our efforts in a way that is more understandable and streamlines our efforts to build a national digital library, instead of obfuscating it.

The current policy plan of the KB is called: "The power of our network". Over the past few years KB is more and more working with a network-state-of-mind as the default. We are quite active in the Dutch Digital Heritage Network where, together with many partners, we are trying to increase significantly the visibility of our (collective) digital collections by making them more usable, e.g. by connecting them as linked (open) data, and by keeping them in a sustainable manner (digital preservation, persistent identifiers, etc.). We play an active role in the public library network, work close together with university libraries and 
participate in international research project like CLARIAH ${ }^{2}$ (Common Lab Research Infrastructure for the Arts and the Humanities).

\section{Status and results}

In 2015, all energy went into factual integration of the four organisations mentioned above. We also started a 'transition programme', targeted at the KB community in general. When the transition diagram was made in 2016, we evolved the transition programme into more focussed actions. We made a few changes to the organisational structure that can be considered as 'overdue maintenance' ('OFP' in the diagram). We started a management development/leadership programme (knobs: management, knowledge \& competences). We are working on vision, strategy and policy plans (knob: strategy) for major domains of expertise within the $\mathrm{KB}$ (digital preservation, metadata, search \& find, content, IT, etc.). We introduced a social intranet to improve on every aspect of internal communication (knob: culture). We are introducing a more modern approach to 'work': result-driven, more flexible, more empowered; using team strength and leaving room for individual growth (knobs: culture, knowledge \& competences). There is still much work to be done, but we can already say: the KB today is quite different from the organisation it was two or three years ago. For the better.

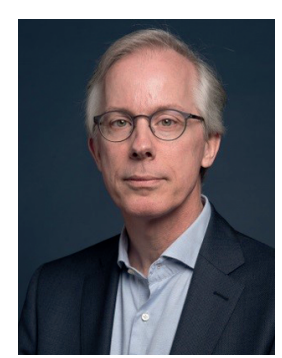

Marc van den Berg

Koninklijke Bibliotheek

Prins Willem-Alexanderhof 5

2595 BE The Hague

The Netherlands

Marc.vandenBerg@KB.nl

2 https://www.clariah.nl. 